J. Phys. IV France 138 (2006) 141-151

(C) EDP Sciences, Les Ulis

DOI: $10.1051 /$ jp4:2006138017

\title{
Génération de supercontinuum dans les fibres optiques microstructurées air-silice : potentialités pour l'émission UV cohérente
}

\author{
P. Leproux ${ }^{1}$, J.L. Auguste ${ }^{1}$, V. Couderc ${ }^{1}$, Ch. Lesvigne ${ }^{1}$, A. Roy ${ }^{1}$, \\ V. Tombelaine ${ }^{1}$, J.M. Blondy ${ }^{1}$, Ph. Roy ${ }^{1}$, C. Restoin ${ }^{1}$ et P.O. Martin ${ }^{1}$ \\ ${ }^{1}$ Institut XLIM, 123 avenue Albert Thomas, 87060 Limoges Cedex, France \\ e-mail : philippe.leproux@xlim.fr ; jean-louis.auguste@xlim.fr
}

\section{INTRODUCTION}

Les fibres microstructurées air-silice (FMAS), apparues dans le milieu des années 90 à l'Université de Southampton [1] (Royaume-Uni), ont démontré un potentiel d'applications extrêmement vaste dans des domaines très variés allant des télécommunications à la biophotonique, en passant par les capteurs ou les sources laser UV. Le laboratoire XLIM (ex-IRCOM, Institut de Recherche en Communications Optiques et Microondes) s'est engagé, depuis 1998, dans la conception, la fabrication et la caractérisation de tels guides de lumière. Nous présentons dans cet article les défis technologiques à relever dans la fabrication des FMAS ainsi qu'une des applications marquantes de ces fibres qu'est la génération de supercontinuum, dans les domaines infrarouge, visible et ultraviolet.

\section{LES DÉFIS TECHNOLOGIQUES DE LA FABRICATION DES FMAS}

\subsection{Le principe du « stack and draw »}

L'un des principaux avantages technologiques des FMAS est de pouvoir s'affranchir d'une étape coûteuse et complexe qu'est la réalisation de la préforme par voie chimique (classiquement obtenue par dopage MCVD). Cependant des développements de procédés ou d'outils spécifiques importants sont nécessaires. Pour répondre à ce défi technologique, le laboratoire a complété son potentiel technologique. En complément de la tour d'étirage de fibres silice utilisée pour la fabrication de fibres hors normes, une tour d'étirage de tubes capillaires et de cannes microstructurées est devenue opérationnelle en 2003. Cet équipement est crucial puisqu'il permet, entre autres, de réaliser les capillaires qui sont les « briques de base élémentaires » nécessaires à la réalisation de la préforme.

Nous allons maintenant détailler le principe de fabrication de ces FMAS ainsi que les contraintes liées à leur réalisation basée sur le principe du « stack and draw », décrit pour la première fois par Knight et al. en 1996 [1]. L'ensemble de la structure hexagonale obtenue (figure 1) par l'assemblage de capillaires d'épaisseur parfois différente est inséré dans un tube afin de maintenir mécaniquement celle-ci. La complexité de cet arrangement réside dans le contrôle précis des tolérances de fabrication de chaque capillaire élémentaire.

Les structures de fibres étudiées sont très différentes les unes des autres et nécessitent des arrangements de tubes capillaires de tailles différentes (essentiellement le diamètre intérieur du capillaire). De plus, la taille des trous (d) souhaitée dans la fibre optique est fréquemment inférieure au micron et le pas $(\Lambda)$ de quelques micromètres. Pour atteindre de telles dimensions, une? opération de fibrage supplémentaire est nécessaire, le rapport de réduction accessible par une seule opération de fibrage n'étant pas suffisant. La phase de fibrage des FMAS pour laquelle le motif (diamètre de trou et pas) est micrométrique va donc se diviser en trois étapes distinctes (figure 2).

La première phase consiste à réaliser la préforme primaire (figure 2a), c'est-à-dire l'arrangement de tous les capillaires au sein d'un tube de maintien. La seconde étape permet de réduire le diamètre de cette 


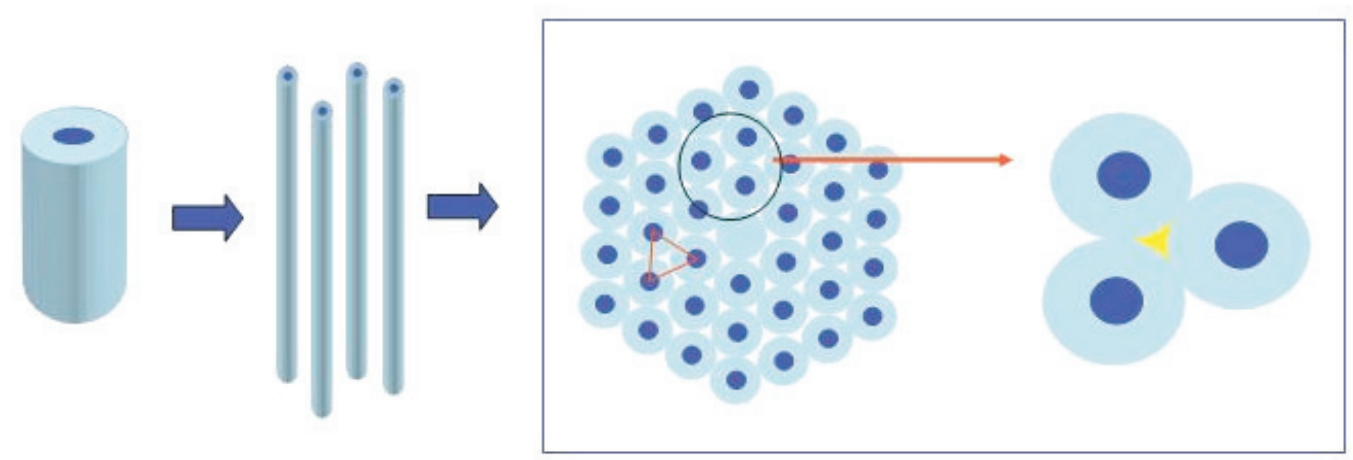

Figure 1. Principe du «stack and draw » et détail de la maille de base triangulaire.
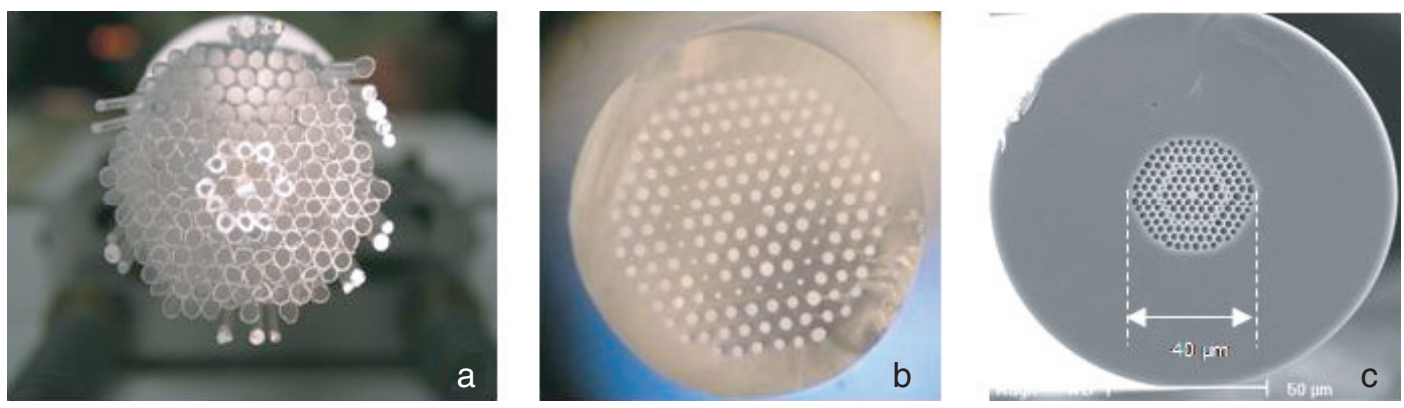

Figure 2. Structures air-silice (a : préforme $(\phi=25 \mathrm{~mm}), \mathrm{b}:$ canne $(\phi=2 \mathrm{~mm})$ et $\mathrm{c}$ : fibre $(\phi=125 \mu \mathrm{m}))$.

préforme d'un facteur variant de 5 à 10 : on obtient alors ce que l'on appelle une canne microstructurée air-silice (figure 2b). Celle-ci est alors manchonnée dans un tube de diamètre intérieur correspondant au diamètre extérieur de la canne et ayant un diamètre extérieur en rapport avec le coefficient de réduction désiré lors de la troisième et ultime étape de fibrage qui conduira à la fibre optique microstructurée (figure 2c).

\subsection{Les contraintes et paramètres de fabrication}

Les contraintes sont multiples et sont essentiellement liées au fait que les matériaux constituant la préforme sont de natures différentes : il s'agit d'air et de verre de silice ultra pure appelé simplement silice par abus de langage.

Une des premières contraintes à gérer est la suppression des trous interstitiels (zone symbolisée en jaune sur la figure 1). Sans précaution particulière lors du fibrage, ces derniers vont se retrouver dans la canne puis dans la fibre optique. Une solution possible consiste à réaliser une dépression entre les capillaires lors de la phase de réalisation de la canne microstructurée. Cette dépression devra être maintenue constante pendant toute la durée du processus.

Un autre paramètre qui devient également critique lors de la réalisation de ces fibres est la température. En effet il ne faut pas perdre de vue que la préforme à fibrer est constituée d'air et de silice, deux matériaux qui n'ont pas le même coefficient de dilatation thermique. Fibrer à une température trop basse entraîne un risque de rupture et des trous interstitiels peuvent subsister, la dépression n'étant plus assez importante pour les refermer. Fibrer à une température trop élevée risque de boucher les trous, la 

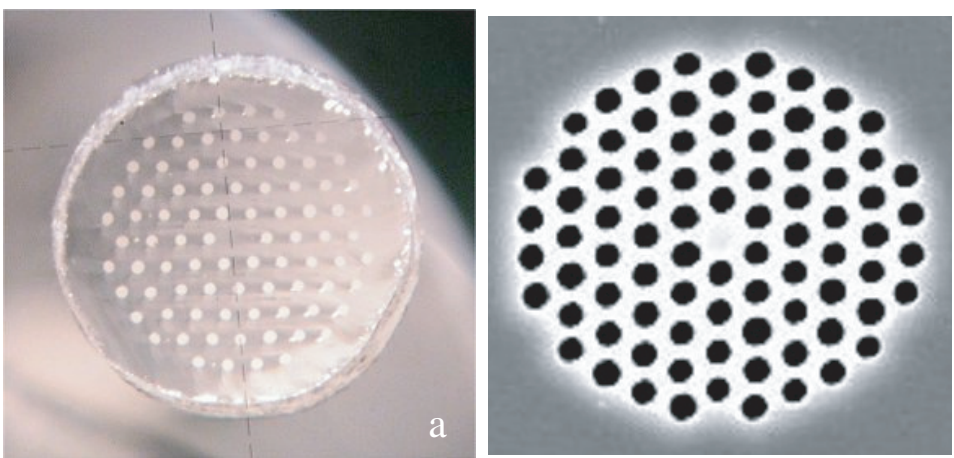

$\mathrm{d}=1,55 \mu \mathrm{m}, \Lambda=2,4 \mu \mathrm{m} \Rightarrow \mathrm{d} / \Lambda=0,65$

$\mathrm{d}=1,4 \mu \mathrm{m}, \Lambda=2 \mu \mathrm{m} \Rightarrow \mathrm{d} / \Lambda=0,7$

$\mathrm{d}=1,9 \mu \mathrm{m}, \Lambda=2,4 \mu \mathrm{m} \Rightarrow \mathrm{d} / \Lambda=0,8$

$\mathrm{d}=2 \mu \mathrm{m}, \Lambda=3,3 \mu \mathrm{m} \Rightarrow \mathrm{d} / \Lambda=0,66$

$\mathrm{d}=4,2 \mu \mathrm{m}, \Lambda=9,5 \mu \mathrm{m} \Rightarrow \mathrm{d} / \Lambda=0,44$

Figure 3. A partir d'une même canne (a) et selon les conditions de température, la fibre obtenue (b) peut présenter des rapports diamètre de trou $(\mathrm{d})$ sur pas $(\Lambda)$ différents.

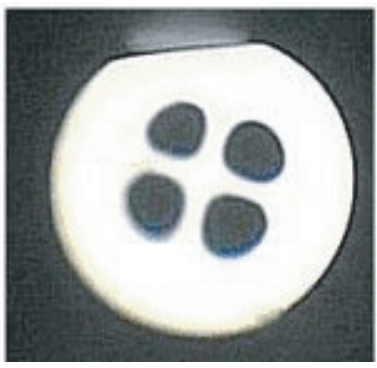

a)

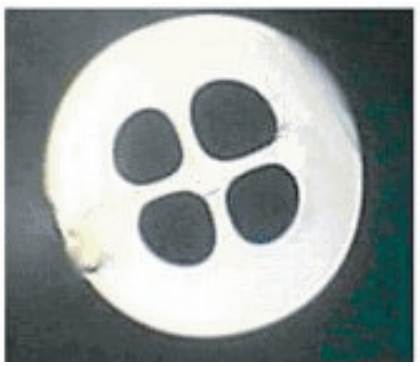

b)

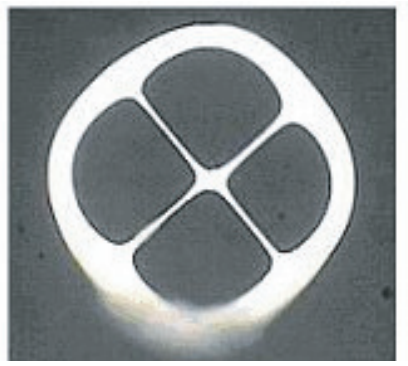

c)

Figure 4. A température de fibrage constante, mise en évidence de l'effet de la pression interne sur la taille des trous dans la fibre optique.

silice à l'intérieur du tube s'écoulant alors dans le capillaire jusqu'à le boucher. La figure 3 montre qu'à partir d'une même canne de départ, et en ajustant finement ce paramètre, on peut obtenir une variation importante des dimensions des trous dans la fibre.

Par ailleurs, à température fixée, le contrôle de la pression interne dans les capillaires permet d'obtenir une dilatation importante, utile à la fabrication de fibres à forte proportion d'air dans la gaine (figure 4).

Deux autres paramètres sont liés et influencent également la qualité de la structure finale : ce sont la vitesse de descente de la préforme dans le four et la vitesse de tirage de la canne ou la vitesse d'enroulement de la fibre. En effet, à un même couple de vitesses correspond un même diamètre extérieur de fibre (ou canne microstructurée), à ceci près que le temps passé dans le four n'est pas le même, ayant pour conséquence une dilatation de l'air dans la préforme différente, et au final une modification de la géométrie de la structure.

Ainsi, on peut mettre en évidence quatre paramètres pertinents de fibrage interdépendants qui sont la pression différentielle dans la préforme, la température de fibrage, les vitesses de descente de la préforme et de fibrage. Pour réaliser une fibre optimale, il faut que celle-ci présente à l'échelle microscopique la même géométrie que celle de la préforme : pour chaque fibre, ce quadruplet de paramètres doit être optimisé et ce de manière précise (à quelques degrés près parfois pour la température de fibrage, autour d'une valeur moyenne d'environ $1800^{\circ} \mathrm{C}$ ). 


\subsection{Les développements actuels}

Aujourd'hui la maîtrise de la fabrication est basée sur l'expérience qui atteint ses limites lorsqu'il est question de fabriquer des structures constituées de matériaux et/ou de géométrie fortement hétérogènes, difficulté sans cesse renouvelée pour chaque type de FMAS en projet.

C'est pourquoi des développements théoriques concernant l'étude du matériau qu'est la silice et les aspects écoulement air-silice liés au fibrage des FMAS sont en cours [2].

Pour ce faire trois axes d'étude ont été définis :

- La modélisation du four à induction (figure 5) permettant, entre autres : de définir la température de la préforme (et non plus une mesure relative faite sur le noyau de carbone), d'optimiser la taille du noyau de graphite en fonction de la préforme à tirer, de quantifier l'influence des feutres de graphite situés de part et d'autre du noyau et d'obtenir le gradient de température longitudinal.

- La simulation de la forme du cône de coulée (passage entre la préforme et le capillaire ou la canne) en relation directe avec la première étape. On a démontré expérimentalement que la forme (longueur essentiellement) du cône de coulée influençait la géométrie transverse de la fibre réalisée.

- L'évolution du rapport air/silice pour un capillaire en fonction des paramètres de fibrage. En effet en contrôlant les paramètres température et vitesses, il est possible à partir d'un même tube de départ d'obtenir des capillaires dont l'épaisseur n'est pas le simple rapport de réduction dû au fibrage. Actuellement les enjeux sont situés autour de la réalisation de capillaires dont le rapport air/silice avoisine les $98 \%$ !
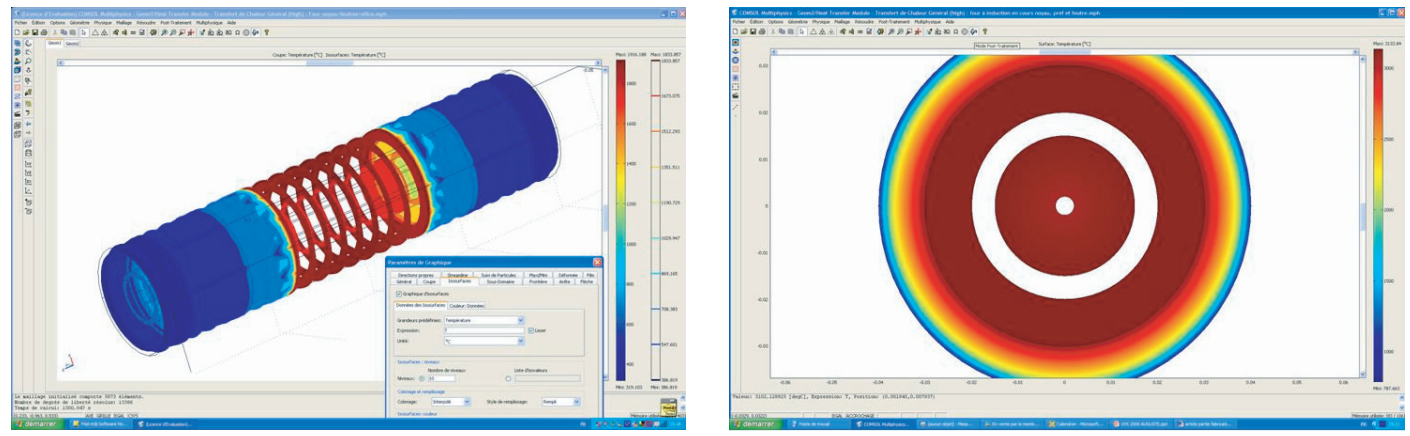

Figure 5. Modélisations par la méthode des éléments finis. A gauche : résultat de la modélisation 3D d'un four à induction. A droite : gradient thermique transverse au sein des différents éléments du four et d'un tube de silice.

Ces premiers résultats ouvrent la voie à des modélisations mettant en jeu différents domaines de la physique (thermique, mécanique, optique), et donnent lieu à des études concernant, par exemple, l'influence d'une variation de température sur la propagation de la lumière (stabilité des capteurs, dérive d'une source laser ...).

En parallèle, d'autres travaux plus expérimentaux sont mis en place pour utiliser d'autres matériaux que le verre de silice pure. Des applications différentes ou aux performances supérieures, inaccessibles actuellement, pourront être proposées, avec par exemple l'utilisation de verres de silice dopée [3] ou l'application de la technologie sol-gel aux FMAS pour la réalisation de couches ultra minces à l'intérieur de FMAS [4].

\section{GÉNÉRATION DE SUPERCONTINUUM DANS LES FMAS}

Ces dernières années, les FMAS associées à des microlasers ont supplanté les chaînes laser lourdes et onéreuses et ont ainsi ouvert la voie à la réalisation de sources laser blanches cohérentes (supercontinua), 
compactes et utilisables dans des appareils portables (outils de diagnostic cellulaire, d'imagerie haute résolution, de spectroscopie ...). En particulier, grâce à une méthode de pompage originale à deux longueurs d'onde, la génération de spectres larges et homogènes a été démontrée à la fois dans les domaines visible et infrarouge (IR). Le même type de résultat a pu être établi en n'utilisant cette fois-ci qu'une seule longueur d'onde de pompe externe, située loin du zéro de dispersion de la fibre. Par ailleurs, l'utilisation de nouveaux designs de FMAS non linéaires a également conduit à l'obtention de résultats originaux et prometteurs en termes de génération de supercontinuum. Une structure rendue fortement biréfringente par l'insertion de deux gros trous près du cœur et une structure à cour dopé Ytterbium $(\mathrm{Yb})$ et à gaine d'air en sont deux exemples détaillés ci-après.

\subsection{Méthode de pompage à deux longueurs d'onde}

Cette méthode proposée depuis 2004 [5] consiste à pomper une FMAS non linéaire au moyen de deux ondes de longueurs d'onde situées de part et d'autre et loin du zéro de dispersion du mode fondamental. De ce fait, on emploie couramment comme source de pompe, pour des raisons de facilité et de compacité, un microlaser Nd:YAG nanoseconde doublé en fréquence émettant à la fois à 532 et 1064 nm (voir montage expérimental schématisé sur la figure 6). La FMAS utilisée présente quant à elle une structure hexagonale à symétrie azimutale $\pi / 3$ (figure 7). Le zéro de dispersion chromatique du mode fondamental de cette fibre est situé à $870 \mathrm{~nm}$.

La technique de pompage à deux longueurs d'onde permet d'obtenir un élargissement spectral important à la fois dans le domaine visible (de 400 à $700 \mathrm{~nm}$, i.e., en régime de dispersion fortement normal) et dans le domaine IR (de 1050 à $1750 \mathrm{~nm}$, i.e., en régime de dispersion anormal), comme le montre la figure 8. L'émission est spatialement monomode (mode fondamental $\mathrm{LP}_{01}$ ) sur l'ensemble du domaine spectral, ce qui laisse entrevoir de nombreuses applications nécessitant une source optique large bande spatialement cohérente. Le mécanisme non linéaire mis en jeu dans la génération de

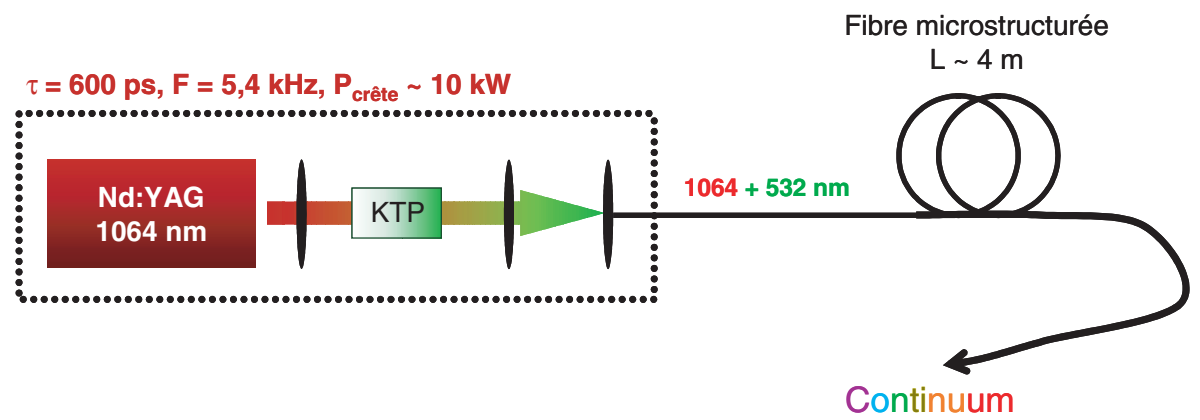

Figure 6. Montage expérimental utilisé pour la technique de pompage à deux longueurs d'onde.

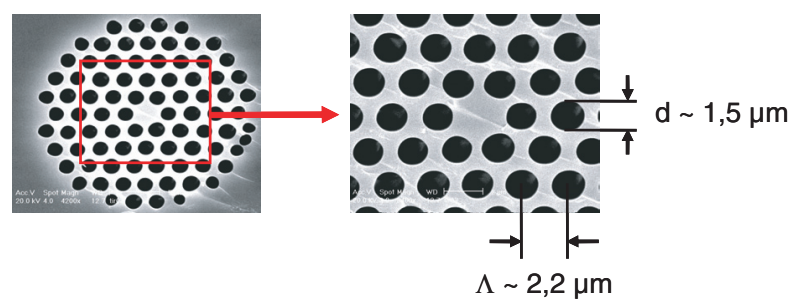

Figure 7. Image MEB du profil transverse de la FMAS utilisée pour la technique de pompage à deux longueurs d'onde. 

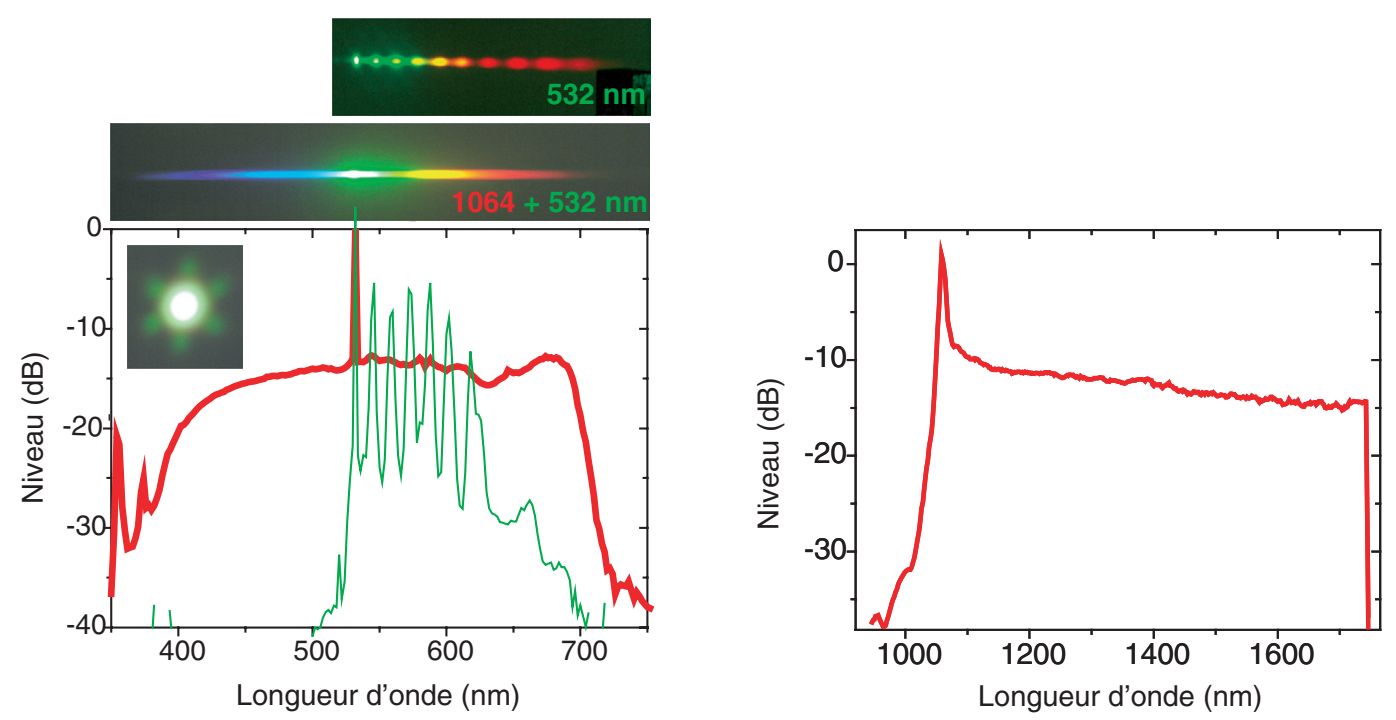

Figure 8. Elargissements spectraux visible et IR obtenus par pompage à deux longueurs d'onde dans une FMAS, avec mise en évidence du faisceau de sortie spatialement monomode (mode $\mathrm{LP}_{01}$ ).

supercontinuum par bi-pompage est aujourd'hui bien identifié (instabilités de modulation puis effets solitoniques avec auto-décalage Raman dans l'IR, modulation de phase croisée dans le visible avec décalage vers le rouge et décalage vers le bleu) [6-7] et devrait permettre de contrôler l'élargissement spectral en fonction des besoins de l'application. Les sources de supercontinuum basées sur la technique de bi-pompage possèdent ainsi un fort potentiel applicatif du fait de leur flexibilité.

Il est à noter que ces démonstrations expérimentales de génération de supercontinuum par pompage à deux longueurs d'onde ont également permis d'établir la disparition complète d'une cascade Raman en régime de dispersion normal [8].

\subsection{Pompage unique éloigné du zéro de dispersion}

La génération de supercontinuum ultra large bande dans une FMAS a également pu être mise en évidence par l'utilisation d'une seule longueur d'onde de pompe (1064 nm), issue d'un microlaser $\mathrm{Nd}$ :YAG nanoseconde et située loin du zéro de dispersion de la fibre (de structure identique à celle montrée précédemment) [9]. Dans ce cas, l'élargissement spectral, de 350 à $1750 \mathrm{~nm}$ (limites de l'analyseur de spectres utilisé), couvre la totalité de la fenêtre de transparence de la silice (voir figure 9). Le mécanisme non linéaire mis en jeu semble être basé sur un mélange à quatre ondes faisant intervenir le premier mode transverse d'ordre supérieur de la fibre $\left(\mathrm{LP}_{11}\right)$. L'émission reste spatialement monomode mais se fait sur ce mode $\mathrm{LP}_{11}$ dans le domaine visible.

\subsection{Utilisation d'une FMAS fortement biréfringente}

La génération de supercontinuum dans les FMAS fortement biréfringentes a déjà fait l'objet de publications [10]. Les instabilités de modulation vectorielles ont plus particulièrement été étudiées de manière numérique et expérimentale [11-12] dans ce type de guide. Dans le but d'améliorer les conditions d'accord de phase du mélange à quatre ondes mentionné dans le paragraphe précédent, une FMAS non linéaire présentant une forte biréfringence de forme a été conçue et fabriquée au laboratoire XLIM. La figure 10 présente la structure retenue, dans laquelle deux trous de taille supérieure ont été 


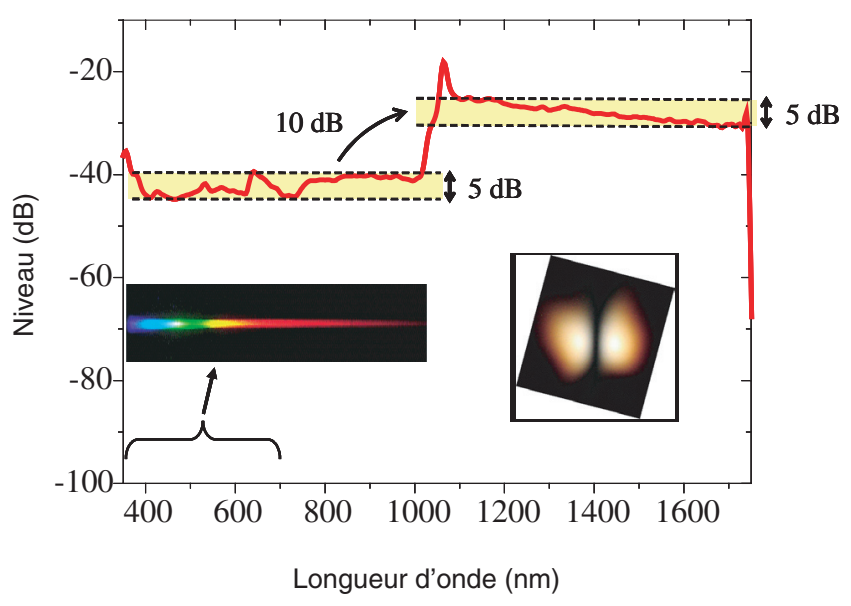

Figure 9. Elargissement spectral obtenu par pompage à une seule longueur d'onde dans une FMAS, avec mise en évidence du faisceau de sortie spatialement monomode (mode $\mathrm{LP}_{11}$ dans le visible).

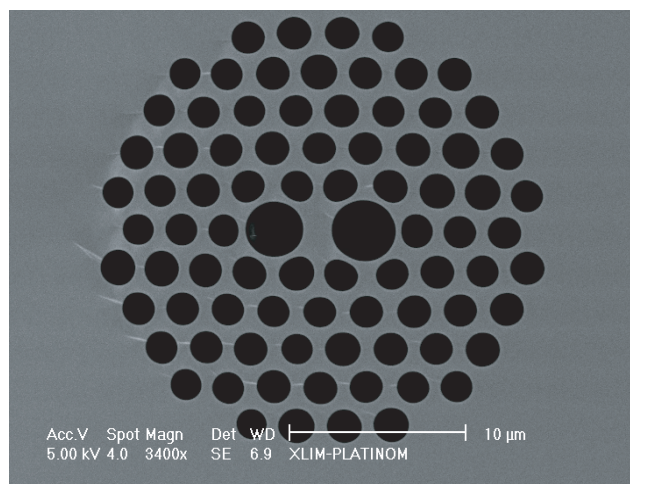

Figure 10. Image MEB du profil transverse de FMAS fortement biréfringente.

insérés près du cœur. La biréfringence de groupe de cette fibre, mesurée à 1,55 $\mu \mathrm{m}$ par la méthode du spectre cannelé, est de l'ordre de $10^{-2}$, valeur particulièrement élevée pour une fibre optique.

Pompée à $1064 \mathrm{~nm}$ dans les mêmes conditions qu'au paragraphe 3.2 et pour une orientation particulière de la polarisation incidente (suivant l'axe des deux gros trous), cette fibre biréfringente donne lieu à la génération du spectre tracé sur la figure 11. Deux pics présents sur ce spectre, respectivement à 830 et $1482 \mathrm{~nm}$, mettent en évidence le développement du supercontinuum à partir d'un mélange à quatre ondes dégénéré et accordé en phase $\left(2 \omega_{1064}=\omega_{830}+\omega_{1482}\right)$ [13]. Comme précédemment, l'élargissement spectral se développe sur le mode $\mathrm{LP}_{11}$ dans le domaine visible. Le mécanisme non linéaire de construction du continuum mis en jeu dans ce cas particulier de FMAS pourrait par la suite être optimisé grâce à l'utilisation d'une structure de fibre adaptée, en vue notamment de relever le niveau du spectre dans le visible.

Par ailleurs, dans le cas de la génération de supercontinuum par pompage unique éloigné du zéro de dispersion a été émise l'hypothèse consistant à dire que le phénomène non linéaire pouvait faire intervenir la création de la seconde pompe $(532 \mathrm{~nm})$ directement dans la FMAS. Pour cette raison, la génération de second harmonique dans la FMAS biréfringente a fait l'objet d'une étude expérimentale. Le niveau de puissance à $532 \mathrm{~nm}$ en sortie de fibre $\left(\mathrm{P}_{532}\right)$ a été mesuré à l'aide d'un photomultiplicateur en fonction du niveau de puissance à $1064 \mathrm{~nm}$ injecté dans la fibre $\left(\mathrm{P}_{1064}\right)$. Les 


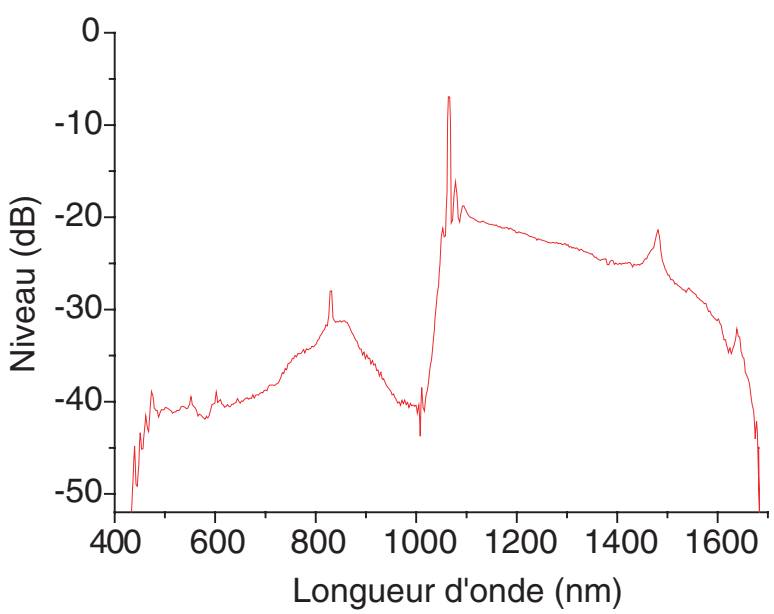

Figure 11. Elargissement spectral obtenu par pompage à une seule longueur d'onde dans une FMAS fortement biréfringente.

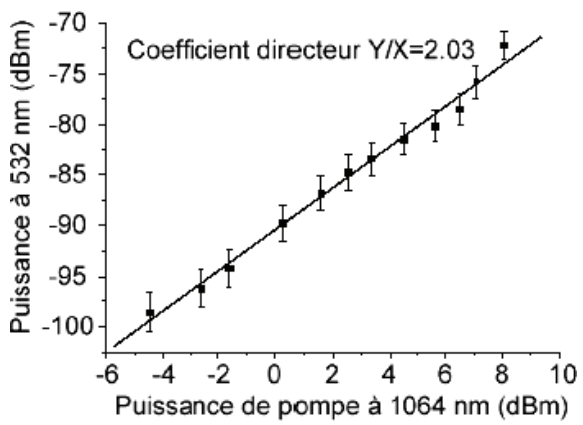

Figure 12. Mesure de la puissance au second harmonique en fonction de la puissance de pompe à $1064 \mathrm{~nm}$ dans une FMAS fortement biréfringente.

résultats obtenus (figure 12) démontrent l'évolution quadratique de $\mathrm{P}_{532}$ en fonction de $\mathrm{P}_{1064}$, ce qui porterait à confirmer l'existence d'un effet de doublage de fréquence au sein même de la fibre [14]. Des mesures complémentaires sont en cours à ce sujet.

\subsection{Utilisation d'une FMAS amplificatrice}

A l'heure actuelle, l'association d'un laser de pompe pulsé (laser à fibre ou microlaser) avec une FMAS non linéaire permet de concevoir des sources de supercontinuum alliant compacité et large bande spectrale, y compris dans le domaine visible. Pour augmenter le niveau global du spectre, une solution consiste à utiliser un laser de pompe de plus forte puissance. Une autre solution, proposée récemment au laboratoire XLIM et ce pour la première fois à notre connaissance, réside dans le fait d'amplifier les impulsions de pompe dans la fibre [15]. Pour cela, cette dernière doit présenter une structure « hybride », dans le sens où elle doit combiner les fonctions « guide non linéaire » et « amplificateur ». La figure 13 montre la FMAS non linéaire et dopée aux ions $\mathrm{Yb}^{3+}$ qui a été fabriquée en vue de jouer ce double rôle. Afin de coupler une forte puissance de pompe multimode des ions $\mathrm{Yb}^{3+}$, un schéma de pompage par la gaine a été retenu, expliquant la présence à la fois d'une gaine interne et d'une gaine externe de type 


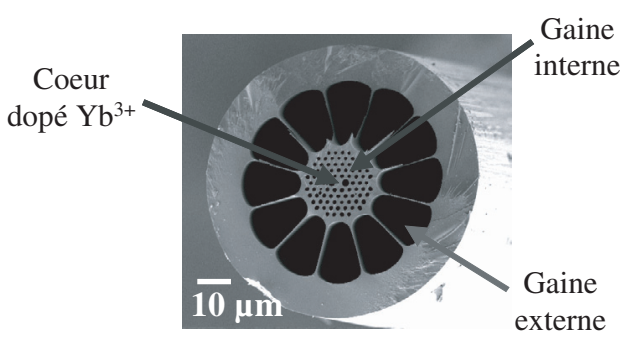

Figure 13. Image MEB du profil transverse de FMAS hybride (non linéaire et amplificatrice).

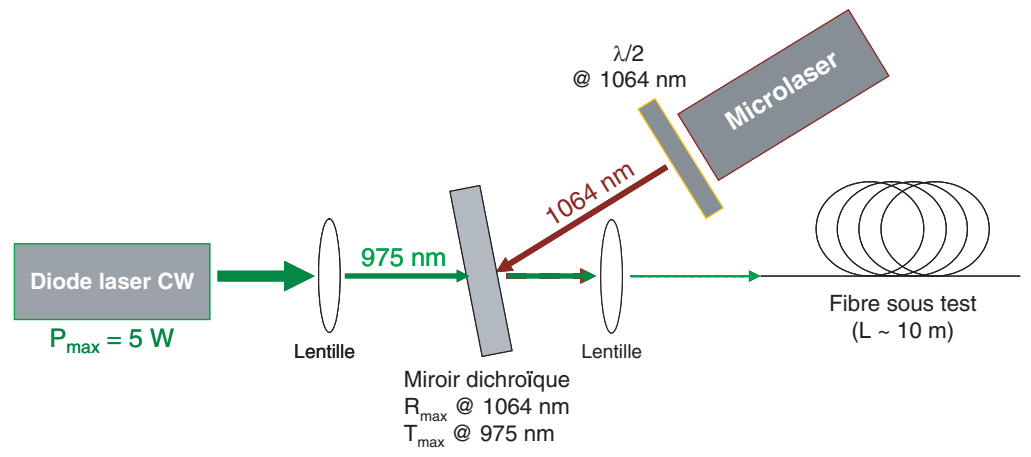

Figure 14. Montage expérimental utilisé pour mettre en œuvre le pompage d'une FMAS hybride (non linéaire et amplificatrice).

« air-clad ». Les paramètres opto-géométriques de la microstructure ont été choisis dans le but d'obtenir un fort coefficient non linéaire dans le cœur et un zéro de dispersion du mode fondamental situé autour de $1 \mu \mathrm{m}$. Cette optimisation a dû être effectuée en tenant compte de l'influence de la proportion d'air de la gaine interne microstructurée sur l'ouverture numérique de cette dernière, dont la valeur définit le rendement d'injection de la pompe multimode.

La FMAS hybride réalisée a été testée en terme de génération de supercontinuum, en fonction du niveau de puissance de pompe multimode $\left(\mathrm{P}_{\text {multi }}\right)$ injectée dans la gaine interne. Pour cela, le faisceau à $1064 \mathrm{~nm}$ issu d'un microlaser Nd:YAG nanoseconde est adressé dans le cœur de la structure, alors qu'une diode multimode et continue émettant à $975 \mathrm{~nm}$ est utilisée pour pomper les ions $\mathrm{Yb}^{3+} \mathrm{du}$ cœur par la gaine (voir montage expérimental sur la figure 14). Les résultats obtenus dans la bande $500-900 \mathrm{~nm}$ (i.e., en régime de dispersion normal), présentés sur la figure 15, montrent que la densité spectrale de puissance du supercontinuum généré augmente avec $\mathrm{P}_{\text {multi }}$. Pour une valeur $\mathrm{P}_{\text {multi }}=1,5 \mathrm{~W}$ seulement, on enregistre un gain moyen d'environ $10 \mathrm{~dB}$ dans la bande spectrale étudiée. De plus, le spectre a tendance à évoluer vers une meilleure platitude. On note également une augmentation du niveau du supercontinuum dans le domaine IR. Enfin, les élargissements spectraux observés se développent sur le mode fondamental $\mathrm{LP}_{01}$. Ce nouveau concept de fibre hybride ouvre une voie intéressante pour le développement de sources blanches cohérentes de forte puissance.

\section{CONCLUSION}

Dans cet article ont tout d'abord été exposés les défis technologiques liés à la fabrication des fibres optiques microstructurées air-silice. De plus, différents résultats expérimentaux ont été présentés, proposant différentes voies pour améliorer et/ou contrôler la génération de supercontinuum dans les fibres microstructurées. Dans tous les cas, les élargissements spectraux obtenus atteignent le domaine 


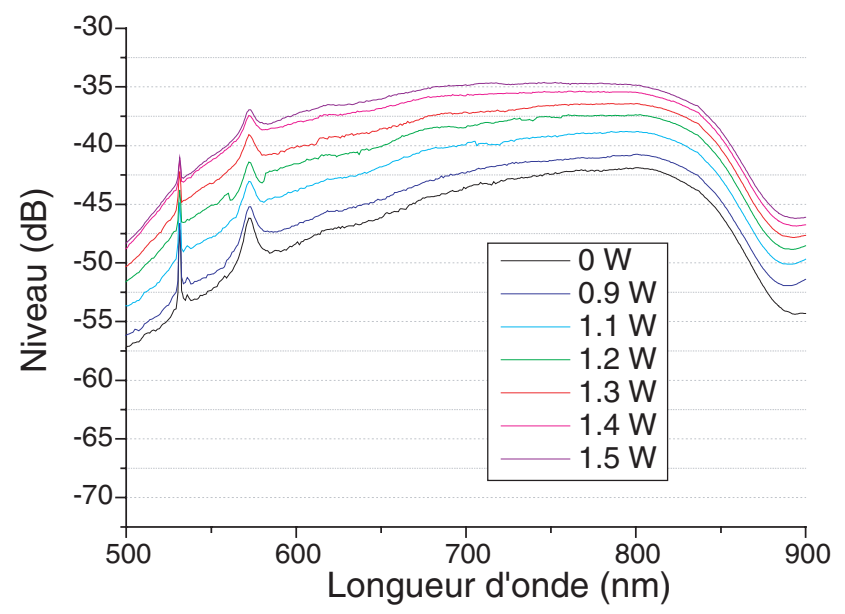

Figure 15. Supercontinua enregistrés en sortie de FMAS hybride pour différentes valeurs de puissance de pompe multimode injectée.

visible jusqu'aux longueurs d'onde bleues, voire jusqu'à la limite basse $(350 \mathrm{~nm})$ des analyseurs de spectres classiquement utilisés pour travailler avec les fibres silice. L'analyse de la génération de continuum en dessous de $350 \mathrm{~nm}$ est en cours, des résultats préliminaires montrant déjà la présence d'énergie à ces courtes longueurs d'onde. Le niveau de puissance attendu est naturellement limité par l'atténuation intrinsèque du matériau, mais de premières réalisations de fibres microstructurées à partir de silices optimisées pour l'UV ont d'ores et déjà donné des résultats prometteurs. Enfin, la recherche de solutions technologiques basées sur de nouveaux matériaux véritablement adaptés au domaine UV doit assurément être poursuivie.

\section{Références}

[1] J. C. Knight et al., "All-silica single-mode optical fiber with photonic crystal cladding," Optics Letters 21, p. 1547-1549, 1996.

[2] J. L. Auguste et al., "Modélisations thermiques pour la fabrication de fibres microstructurées air-silice," soumis aux JNOG 2006, Metz.

[3] J. L. Auguste et al., "Développements technologiques pour l'élaboration de fibres « verres silice »" soumis aux JNOG 2006, Metz.

[4] C. Restoin et al., "Technique sol gel appliquée à la réalisation de fibres à coeur creux," JNOG 2005, Chambéry.

[5] P. A. Champert et al., "White-light supercontinuum generation in normally dispersive optical fiber using original multi-wavelength pumping system," Optics Express 12, p. 4366-4371, 2004.

[6] G. Genty et al., "Route to broadband blue-light generation in microstructured optical fibers," Optics Letters. 30, p. 756-758, 2005.

[7] T. Schreiber et al., "Supercontinuum generation by femtosecond single and dual wavelength pumping in photonic crystal fibers with two zero dispersion wavelengths," Optics Express 13, p. 9556-9569, 2005.

[8] V. Couderc et al., "Raman cascade suppression by using wide band parametric conversion in large normal dispersion regime," Optics Express 13, p. 8584-8590, 2005.

[9] V. Tombelaine et al., "Ultra wide band supercontinuum generation in air-silica holey fibers by SHG-induced modulation instabilities," Optics Express 13, p. 7399-7404, 2005. 
[10] M. Lehtonen et al., "Supercontinuum generation in a highly birefringent microstructured fiber," Applied Physics Letters 82, p. 2197-2199, 2003.

[11] G. Millot et al., "Polarization mode dispersion and vectorial modulational instability in air-silica microstructure fiber," Optics Letters 27, p. 695-697, 2002.

[12] A. Tonello et al., "Frequency tunable polarization and intermodal modulation instability in high birefringence holey fiber," Optics Express 14, p. 397-404, 2005.

[13] C. Lesvigne et al., "Second and third order nonlinearities in a highly birefringent holey fiber for super-continuum generation," CLEO 2006, Long Beach, California, USA.

[14] C. Lesvigne et al., "Second Harmonic Generation in a Highly Birefringent Nonlinear Microstructured Fibre," ECOC 2006, Cannes, France.

[15] A. Roy et al., "Double-clad nonlinear microstructured fiber for white laser applications," CLEO 2006, Long Beach, California, USA. 